\title{
Cycle Slip Detection and Correction for Heading Determination with Low-Cost GPS/INS Receivers
}

\author{
Patrick Henkel and Naoya Oku
}

\begin{abstract}
Precise attitude determination with low-cost GPS receivers requires integer ambiguity resolution and reliable cycle slip correction. In this paper, a tree search of cycle slips is proposed, which combines double difference GPS carrier phases from all visible satellites, gyroscope and acceleration measurements, and a priori information on the baseline length between both GPS receivers. The proposed method was verified in both a slalom drive with high vehicle dynamics and a drive below trees with shadowed GPS signals: The residuals of the fixed phase measurements were reduced to less than $15 \mathrm{~cm}$ throughout the measurement period.
\end{abstract}

\section{Keywords}

Attitude determination • Cycle slip correction • Integer ambiguity resolution

\section{Introduction}

The availability of mass-market GPS receivers with carrier phase tracking has led to a wide range of new applications of RTK and attitude determination. However, the measurements of low-cost GPS receivers differ in three aspects from the measurements of geodetic receivers: First, code multipath is much larger due to the small size of the patch antennas. It can be $10 \mathrm{~m}$ even in open-sky conditions, which is a challenge for ambiguity resolution. Secondly, half cycle slips occur much more frequently and at multiple satellites simultaneously. Today, cycle slip detection and correction can be performed reliably for geodetic receivers with inertial sensors: Du and Gao (2012) differenced the carrier phase measurements between two satellites and two subsequent epochs such that clock offsets, ambiguities, biases and

P. Henkel $(\square)$

Technische Universität München, Theresienstrasse 90, 80333

München, Germany

e-mail: patrick.henkel@tum.de

N. Oku

Advanced Navigation Solutions - ANAVS, Friedrichshafener Str. 1, 82205 Gilching, Germany atmospheric delays are eliminated (except for the drift which is in general negligible for periods of less than $1 \mathrm{~s}$ ). This leaves the change in position and the cycle slips as unknowns. The change in position is predicted by an inertial sensor. Thus, cycle slips can be determined on a satellite by satellite basis by simple rounding. Du and Gao (2012) applied a cascaded approach to two dual-frequency Novatel OEM4 receivers, i.e. the widelane cycle slips were determined first. Subsequently, the extra-widelane cycle slips were resolved and, finally, the L1 and L2 cycle slips were derived from the widelane and extra-widelane cycle slips.

Dai et al (2009) proposed a cycle slip detection and correction method for triple frequency GNSS receivers. They used two triple frequency geometry-free phase combinations and performed an integer least-squares estimation using the LAMBDA method of Teunissen (1995).

Colombo et al (1999) also proposed a cascaded cycle slip detection for dual frequency receivers. The widelane cycle slips were fixed as described by Du and Gao (2012). The individual L1 and L2 cycle slips were then directly fixed with the help of the widelane cycle slips. They used an Ashtech receiver and high-grade IMU with a gyroscope drift of only $3^{\circ} / \mathrm{h}$, and were able to correct $99.1 \%$ of cycle slips for data gaps of $5 \mathrm{~s}$. 
Fig. 1 Residuals of fixed phase residuals are clearly smaller than the wavelength and are drift-free, which indicates a correct integer ambiguity resolution. The biases of up to $2 \mathrm{~cm}$ arise from a small bias in the baseline length constraint due to antenna phase center offsets solution during initialization: the

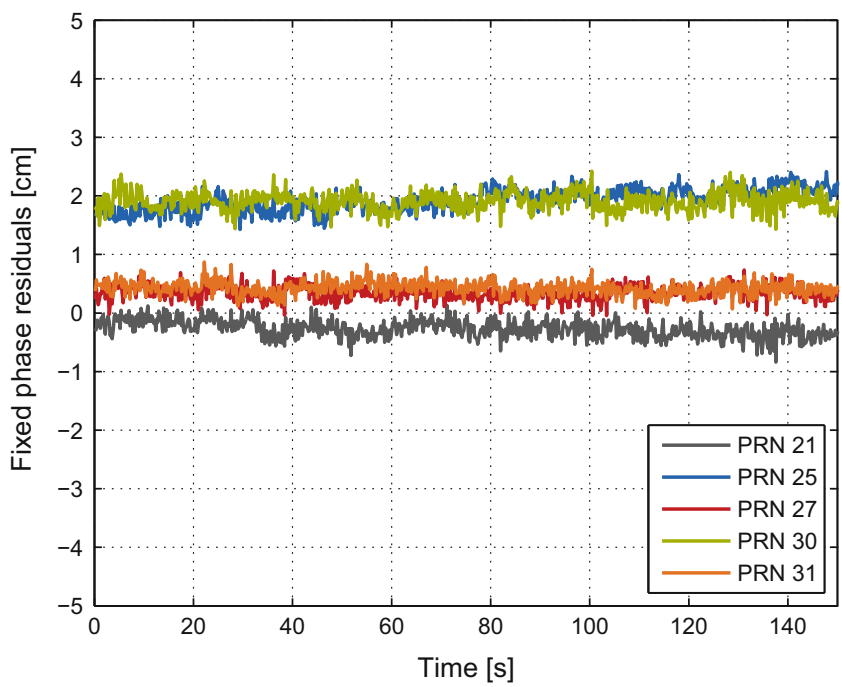

These methods can not be used for low-cost singlefrequency mass-market GNSS receivers and inertial sensors: The receivers show half cycle slips, i.e. jumps of integer multiples of $\lambda / 2=9.5 \mathrm{~cm}$, and substantial phase and code multipath. The measurement model must be strengthened, e.g. by including baseline a priori information as described by Henkel and Kiam (2013).

A third difference between low-cost and geodetic GNSS receivers is that the receiver clock offsets are in the order of milliseconds instead of nanoseconds (see Henkel and Gunther 2013), which implies that the satellite movement within the time span of the differential receiver clock offset is no longer negligible. A synchronization correction is required to preserve the integer property of the double difference (DD) ambiguities.

Therefore, we include the receiver clock offset explicitly in our model for the DD carrier phase measurements. As the baseline is short for attitude determination, atmospheric and orbital errors can be neglected and we obtain

$$
\begin{aligned}
& \left(\lambda \varphi_{1}^{k}\left(t+\delta \tau_{1}\right)-\lambda \varphi_{1}^{l}\left(t+\delta \tau_{1}\right)\right) \\
& -\left(\lambda \varphi_{2}^{k}\left(t+\delta \tau_{2}\right)-\lambda \varphi_{2}^{l}\left(t+\delta \tau_{2}\right)\right) \\
& =\left\|x_{1}\left(t+\delta \tau_{1}\right)-x^{k}\left(t+\delta \tau_{1}-\Delta \tau_{1}^{k}\right)\right\| \\
& \quad-\left\|x_{1}\left(t+\delta \tau_{1}\right)-x^{l}\left(t+\delta \tau_{1}-\Delta \tau_{1}^{l}\right)\right\| \\
& \quad-\left\|x_{2}\left(t+\delta \tau_{2}\right)-x^{k}\left(t+\delta \tau_{2}-\Delta \tau_{2}^{k}\right)\right\| \\
& \quad+\left\|x_{2}\left(t+\delta \tau_{2}\right)-x^{l}\left(t+\delta \tau_{2}-\Delta \tau_{2}^{l}\right)\right\| \\
& \quad+\lambda N_{12}^{k l}+\lambda / 2 \Delta N_{12}^{k l}(t)+m_{\varphi_{12}^{k l}}\left(t+\delta \tau_{1}, t+\delta \tau_{2}\right) \\
& \quad+\varepsilon_{\varphi_{12}^{k l}}\left(t+\delta \tau_{1}, t+\delta \tau_{2}\right),
\end{aligned}
$$

with the carrier wavelength $\lambda$, the undifferenced phase measurement $\varphi_{r}^{k}$ of receiver $r$ and satellite $k$, the receiver clock offset $\delta \tau_{r}$, the receiver position $x_{r}$, the satellite position $x^{k}$, the DD integer ambiguity $N_{12}^{k l}$, the DD half cycle slip $\Delta N_{12}^{k l}$, the DD phase multipath $m_{\varphi_{12}^{k l}}$ and the DD phase noise $\varepsilon_{\varphi_{12}^{k l}}$. The synchronization correction is given by

$$
\begin{aligned}
\lambda \Delta & \varphi_{12}^{k l}\left(t+\delta \tau_{1}, t+\delta \tau_{2}\right) \\
= & \left(e_{1}^{k}\left(t+\delta \tau_{1}\right)\right)^{\mathrm{T}}\left(x_{1}\left(t+\delta \tau_{1}\right)-x^{k}\left(t+\delta \tau_{1}-\Delta \tau_{1}^{k}\right)\right) \\
& -\left(e_{1}^{l}\left(t+\delta \tau_{1}\right)\right)^{\mathrm{T}}\left(x_{1}\left(t+\delta \tau_{1}\right)-x^{l}\left(t+\delta \tau_{1}-\Delta \tau_{1}^{l}\right)\right) \\
& -\left(e_{1}^{k}\left(t+\delta \tau_{2}\right)\right)^{\mathrm{T}}\left(x_{1}\left(t+\delta \tau_{2}\right)-x^{k}\left(t+\delta \tau_{2}-\Delta \tau_{2}^{k}\right)\right) \\
& +\left(e_{1}^{l}\left(t+\delta \tau_{2}\right)\right)^{\mathrm{T}}\left(x_{1}\left(t+\delta \tau_{2}\right)-x^{l}\left(t+\delta \tau_{2}-\Delta \tau_{2}^{l}\right)\right) .
\end{aligned}
$$

We linearize the norms in (1), apply the synchronization correction of (2) and a cycle slip correction to write the linearized corrected DD phase and code measurements in matrix-vector notation as

$$
\left(\begin{array}{c}
\lambda \varphi_{12} \\
\rho_{12}
\end{array}\right)=H b_{12}+A N_{12}+\left(\begin{array}{c}
m_{\varphi_{12}} \\
m_{\rho_{12}}
\end{array}\right)+\left(\begin{array}{c}
\varepsilon_{\varphi_{12}} \\
\varepsilon_{\rho_{12}}
\end{array}\right)
$$

with $H$ and $A$ being the implicitly defined DD geometry and ambiguity coefficient matrices, and $b_{12}$ being the baseline between both receivers. The integer least-squares estimation of the DD integer ambiguities and baseline coordinates is improved by some a priori information $l$ on the baseline length, which leads to the constrained integer least-squares estimation problem:

$$
\begin{gathered}
\min _{b_{12} \in \mathbb{R}^{3 \times 1}, N_{12} \in \mathbb{Z}^{K \times 1}}\left\|\left(\begin{array}{c}
\lambda \varphi_{12} \\
\rho_{12}
\end{array}\right)-H b_{12}-A N_{12}\right\|_{\Sigma_{\varphi_{12}}^{-1}}^{2} \\
\text { s.t. } \quad\left\|b_{12}\right\| \stackrel{!}{=} l .
\end{gathered}
$$

A solution to this problem was developed in Teunissen (2006, 2010). Figure 1 shows the fixed phase residuals of this 
estimator for DD phase measurements from two u-blox LEA 6T GPS receivers mounted on the roof of a car. The residuals are clearly smaller than the wavelength and are drift-free, which indicates a correct integer ambiguity resolution. The biases of up to $2 \mathrm{~cm}$ arise most likely from a bias in the baseline length constraint due to antenna phase center offsets.

\section{Initial Calibration of Inertial Sensors}

We start with a rough alignment by transforming the measured acceleration $a^{\mathrm{s}}$ and angular rotation rate $\omega^{\mathrm{s}}$ from the sensor-fixed (s-) frame to the body-fixed (b-) frame (aligned with longitudinal and transversal axis of car), i.e.

$$
\begin{aligned}
& a_{\text {rough }}^{\mathrm{b}}=C_{\mathrm{s}}^{\mathrm{b}} a^{\mathrm{s}}=R_{1}\left(\phi_{\mathrm{s}}^{\mathrm{b}}\right) R_{2}\left(\theta_{\mathrm{s}}^{\mathrm{b}}\right) R_{3}\left(\psi_{\mathrm{s}}^{\mathrm{b}}\right) a^{\mathrm{s}} \\
& \omega_{\text {rough }}^{\mathrm{b}}=C_{\mathrm{s}}^{\mathrm{b}} \omega^{\mathrm{s}}=R_{1}\left(\phi_{\mathrm{s}}^{\mathrm{b}}\right) R_{2}\left(\theta_{\mathrm{s}}^{\mathrm{b}}\right) R_{3}\left(\psi_{\mathrm{s}}^{\mathrm{b}}\right) \omega^{\mathrm{s}},
\end{aligned}
$$

where the roll angle $\phi_{\mathrm{s}}^{\mathrm{b}}$, the pitch angle $\theta_{\mathrm{s}}^{\mathrm{b}}$ and the yaw angle $\psi_{\mathrm{s}}^{\mathrm{b}}$ are approximated from the mounting of the sensor on the body and $R_{i}$ is the rotation around the $i$-th axis. Subsequently, we average $\omega_{\text {rough }}^{\mathrm{b}}$ in static conditions to determine the biases $b_{\omega}^{\mathrm{b}}$, which are then subtracted from the measurements:

$$
\omega^{\mathrm{b}}=\omega_{\text {rough }}^{\mathrm{b}}-b_{\omega}^{\mathrm{b}}
$$

The acceleration measurements are also averaged over time in static conditions to reduce the noise. The obtained $\bar{a}^{\text {b }}$ is expressed in terms of the Euler angles $\phi, \theta, \psi$ and the gravitational acceleration $g$, i.e.

$$
\begin{aligned}
\bar{a}^{\mathrm{b}} & =C_{\mathrm{n}}^{\mathrm{b}} \bar{a}^{\mathrm{n}} \approx R_{1}(\phi) R_{2}(\theta) R_{3}(\psi)(0,0, g)^{\mathrm{T}}+b_{\bar{a}^{\mathrm{b}}}+\eta_{\bar{a}^{\mathrm{b}}} \\
& =g \cdot(-\sin \theta, \cos \theta \sin \phi, \cos \theta \cos \phi)^{\mathrm{T}}+b_{\bar{a}^{\mathrm{b}}}+\eta_{\bar{a}^{\mathrm{b}}},
\end{aligned}
$$

with the bias $b_{\bar{a}^{\mathrm{b}}}$ and noise $\eta_{\bar{a}^{\mathrm{b}}}$. The biases and misalignment errors $\Delta \theta$ and $\Delta \phi$ between GPS and INS sensors are determined by least-squares estimation once the car is moving, i.e.

$$
\min _{\Delta \theta, \Delta \phi, b_{\bar{a}^{\mathrm{b}}}}\left\|\bar{a}^{\mathrm{b}}-g \cdot\left(\begin{array}{c}
-\sin (\theta+\Delta \theta) \\
\cos (\theta+\Delta \theta) \sin (\phi+\Delta \phi) \\
\cos (\theta+\Delta \theta) \cos (\phi+\Delta \phi)
\end{array}\right)-b_{\bar{a}^{\mathrm{b}}}\right\|^{2}
$$

The roll and pitch angles can then be derived from (7) without the need of knowing $g$ as

$$
\begin{aligned}
& \phi=\operatorname{atan}\left(\left(\bar{a}_{y}^{\mathrm{b}}-b_{\bar{a}_{y}^{\mathrm{b}}}\right) /\left(\bar{a}_{z}^{\mathrm{b}}-b_{\bar{a}_{z}^{\mathrm{b}}}\right)\right) \\
& \theta=\operatorname{atan}\left(-\left(\bar{a}_{x}^{\mathrm{b}}-b_{\bar{a}_{x}^{\mathrm{b}}}\right) / \sqrt{\left(\bar{a}_{y}^{\mathrm{b}}-b_{\bar{a}_{y}^{\mathrm{b}}}\right)^{2}+\left(\bar{a}_{z}^{\mathrm{b}}-b_{\bar{a}_{z}^{\mathrm{b}}}\right)^{2}}\right)
\end{aligned}
$$

We initialize the heading from the GPS fixed solution. Once the Euler angles and a rough estimate of the absolute position (longitude $\lambda$, latitude $\varphi$ ) is available from GPS, the coordinate frame transformation from the $b$-frame to the ECEF e-frame is determined as

$$
C_{\mathrm{b}}^{\mathrm{e}}=C_{\mathrm{n}}^{\mathrm{e}} C_{\mathrm{b}}^{\mathrm{n}}
$$

with

$$
C_{\mathrm{n}}^{\mathrm{e}}=\left(\begin{array}{ccc}
-\sin \varphi \cos \lambda & -\sin \lambda & -\cos \varphi \cos \lambda \\
-\sin \varphi \sin \lambda & \cos \lambda & -\cos \varphi \sin \lambda \\
\cos \varphi & 0 & -\sin \varphi
\end{array}\right)
$$

and $C_{\mathrm{b}}^{\mathrm{n}}=\left(C_{\mathrm{n}}^{\mathrm{b}}\right)^{-1}=\left(R_{1}(\phi) R_{2}(\theta) R_{3}(\psi)\right)^{-1}$. The rotation matrix $C_{\mathrm{e}}^{\mathrm{b}}=\left(C_{\mathrm{b}}^{\mathrm{e}}\right)^{-1}$ is then transformed to a Quaternion as described in Jekeli (2001):

$$
q=\frac{1}{\left\|\left[q_{a}, q_{b}, q_{c}, q_{d}\right]\right\|} \cdot\left[q_{a}, q_{b}, q_{c}, q_{d}\right]^{\mathrm{T}}
$$

with the four quaternion elements $q_{a}, q_{b}, q_{c}$ and $q_{d}$.

\section{Integration of Orientation with Quaternions}

Jekeli (2001) derived the time-derivative of $C_{\mathrm{e}}^{\mathrm{b}}$ as

$$
\dot{C}_{\mathrm{e}}^{\mathrm{b}}=C_{\mathrm{e}}^{\mathrm{b}} \Omega_{\mathrm{be}}^{\mathrm{e}}
$$

which represents a differential equation with unknown $C_{\mathrm{e}}^{\mathrm{b}}$. The skew-symmetric matrix $\Omega_{\text {be }}^{\mathrm{e}}$ is given by

$$
\Omega_{\mathrm{be}}^{\mathrm{e}}=\left(\begin{array}{rrr}
0 & -\omega_{3} & \omega_{2} \\
\omega_{3} & 0 & -\omega_{1} \\
-\omega_{2} & \omega_{1} & 0
\end{array}\right)
$$

where the angular rotation rates $\omega_{i}$ of the e-frame w.r.t. the b-frame are obtained from (6) by subtracting the earth rotation rate, i.e.

$$
\left(\omega_{1}, \omega_{2}, \omega_{3}\right)^{\mathrm{T}}=\omega^{\mathrm{b}}-C_{\mathrm{e}}^{\mathrm{b}} \cdot\left(0,0, \omega_{\mathrm{E}}\right)^{\mathrm{T}}=: \omega_{\mathrm{be}}^{\mathrm{b}} \cdot
$$

The differential equation of (13) shall be solved with Quaternions. Jekeli (2001) transformed the $3 \times 3$ matrix equation of (13) to the $4 \times 1$ vector equation

$$
\dot{q}=\frac{1}{2} A_{q} q
$$

with the quaternion $q$ and the matrix of angular velocities $A_{q}$. The latter one is given by 


$$
A_{q}=\left(\begin{array}{rrrr}
0 & \omega_{1} & \omega_{2} & \omega_{3} \\
-\omega_{1} & 0 & \omega_{3} & -\omega_{2} \\
-\omega_{2} & -\omega_{3} & 0 & \omega_{1} \\
-\omega_{3} & \omega_{2} & -\omega_{1} & 0
\end{array}\right)
$$

We performed the integration of the Quaternion with the third order Runge-Kutta method (see Jekeli 2001), i.e. the Quaternion at time $t_{n+1}$ is given by

$$
\begin{aligned}
q\left(t_{n+1}\right) & =q\left(t_{n}+h\right) \\
& =q\left(t_{n}\right)+h \cdot\left(\frac{1}{6} \delta q_{0}+\frac{2}{3} \delta q_{1}+\frac{1}{6} \delta q_{2}\right),
\end{aligned}
$$

where $h=2 \delta t$ denotes the integration time and $\delta q_{0}, \delta q_{1}$ and $\delta q_{2}$ denote the coefficients given by

$$
\begin{aligned}
& \delta q_{0}=\frac{1}{2} A_{q}\left(t_{n}\right) q\left(t_{n}\right) \\
& \delta q_{1}=\frac{1}{2} A_{q}\left(t_{n}+\frac{h}{2}\right)\left(q\left(t_{n}\right)+h / 2 \delta q_{0}\right) \\
& \delta q_{2}=\frac{1}{2} A_{q}\left(t_{n}+h\right)\left(q\left(t_{n}\right)-h \delta q_{0}+2 h \delta q_{1}\right) .
\end{aligned}
$$

Once the integrated quaternion is determined, it is transformed back to a rotation matrix.

\section{$4 \quad$ Cycle Slip Detection and Correction}

The fixed double DD carrier phase measurement for satellite pair $\{k, l\}$ is modeled as

$$
\lambda\left(\varphi_{12}^{k l}-\check{N}_{12}^{k l}\right)=e^{k l} b_{12}+\lambda / 2 \Delta N_{12}^{k l}+\varepsilon_{12}^{k l},
$$

with the single difference $e^{k l}$ between the unit vectors pointing from the satellites to the receiver, the baseline vector $b_{12}$ between both receivers, the carrier wavelength $\lambda$, the fixed DD integer ambiguity $N_{12}^{k l}$, the unknown DD cycle slip (CS) $\Delta N_{12}^{k l}$ and the DD phase noise $\varepsilon_{12}^{k l}$. Solving (20) for $\Delta N_{12}^{k l}$ yields

$$
\Delta \check{N}_{12}^{k l}=\left[\frac{1}{\lambda / 2}\left(\lambda\left(\varphi_{12}^{k l}-\check{N}_{12}^{k l}\right)-e^{k l} \hat{b}_{12}^{\mathrm{IMU}}\right)\right],
$$

where $[\cdot]$ denotes the rounding operator and $\hat{b}_{\text {IMU }}$ denotes the baseline estimate of the IMU that was obtained in four steps: First, the IMU was initialized with the validated GPS attitude solution of the last GPS measurement epoch. Subsequently, the orientation was integrated using IMU measurements between the epochs of the last and current GPS measurement. The integration was performed with quaternions as described in the previous section. In a third step, the quaternions were transformed to Euler angles and, finally, the horizontal baseline estimate was obtained from the heading $\psi_{\text {IMU }}$ and baseline length a priori information $l$ as

$$
\hat{b}_{12}^{\mathrm{IMU}}=l \cdot\left(\sin \left(\psi_{\mathrm{IMU}}\right), \cos \left(\psi_{\mathrm{IMU}}\right)\right)^{\mathrm{T}} .
$$

The cycle slip correction of (21) might be erroneous if the initial calibration of the IMU was erroneous.

In this case, the cycle slip correction (CSC) can be improved by jointly estimating the CSC and baseline coordinates using fixed DD phase measurements from all available satellites, the IMU baseline estimate of (22) and the baseline length a priori information, i.e.

$$
\min _{b_{12}, \Delta N_{12}}\left\|z_{12}-H b_{12}-A \Delta N_{12}\right\|_{\Sigma_{\varphi}^{-1}}^{2} \quad \text { s.t. } \quad\left\|b_{12}\right\|=l,
$$

with the combined GPS/INS measurement vector

$$
z_{12}=\left(\begin{array}{c}
\lambda\left(\varphi_{12}-\check{N}_{12}\right) \\
\hat{b}_{12}^{\mathrm{IMU}}
\end{array}\right)
$$

and $H$ being the redefined $(K+3) \times 3$ DD geometry matrix, $A(\lambda / 2)$ is the extended $(K+3) \times q$ CSC coefficient matrix for $q$ DD cycle slips, and $l$ describes the a priori information on the baseline length. The minimization of (23) includes a search of $\Delta N_{12}$ inside a predefined search space volume $\chi^{2}$ and an iterative computation of $b_{12}$ for each integer candidate vector $\Delta N_{12}$. We use the orthogonal decomposition of Teunissen (1995) to rewrite the sum of squared errors of (23) as

$$
\begin{aligned}
\| z_{12} & -H b_{12}-A \Delta N_{12} \|_{\Sigma_{z_{12}}^{-1}}^{2} \\
= & \left\|\Delta \hat{N}_{12}-\Delta N_{12}\right\|_{\Sigma_{\hat{N}_{12}^{-1}}^{-1}}^{2}+\left\|\breve{b}_{12}\left(\Delta N_{12}\right)-b_{12}\right\|_{\Sigma_{b_{12}}^{-1}}^{2} \\
& +\left\|P_{\bar{A}}^{\perp} P_{H}^{\perp} z_{12}\right\|_{\Sigma_{z_{12}}^{-1}}^{2},
\end{aligned}
$$

with $P_{H}^{\perp}$ being the projector on the orthogonal complement of the range space of $H$ and $\bar{A}=P_{H}^{\perp} A$. The first term on the right side was further developed by Teunissen (1995):

$$
\left\|\Delta \hat{N}_{12}-\Delta N_{12}\right\|_{\Sigma_{\Delta \hat{N}_{12}}^{-1}}^{2}=\sum_{l=1}^{k} \frac{\left(\Delta N_{12}^{l}-\Delta \hat{N}_{12}^{l \mid 1, \ldots, l-1}\right)^{2}}{\left(\sigma_{\Delta \hat{N}_{12}^{l \mid 1, \ldots, l-1}}\right)^{2}},
$$

with $\Delta \hat{N}_{12}^{l \mid 1, \ldots, l-1}$ being the $l$-th conditional cycle slip estimate. Setting (26) into (25), defining the search space volume $\chi^{2}$ as an upper bound on (25), adding the baseline length 
constraint as a zero term, and solving for the $k$-th ambiguity yields:

$$
\begin{aligned}
& \frac{\left(\Delta N_{12}^{k}-\Delta \hat{N}_{12}^{k \mid 1, \ldots, k-1}\right)^{2}}{\sigma_{\Delta \hat{N}_{12}^{k \mid 1, \ldots, k-1}}^{2}} \leq \chi^{2}-\left\|P_{\bar{A}}^{\perp} P_{H}^{\perp} z_{12}\right\|_{\Sigma_{z_{12}}^{-1}}^{2} \\
& \quad-\sum_{l=1}^{k-1} \frac{\left(\Delta N_{12}^{l}-\Delta \hat{N}_{12}^{l \mid 1, \ldots, l-1}\right)^{2}}{\left(\sigma_{\left.\Delta \hat{N}_{12}^{l 1, \ldots, l-1}\right)^{2}}^{l \mid l}\right.} \\
& -\min _{b_{12}, \mu}\left(\left\|\check{b}_{12}\left(N_{12}\right)-b_{12}\right\|_{\Sigma_{\breve{b}_{12}}^{-1}}^{2}+\mu \cdot\left(\left\|b_{12}\right\|^{2}-l^{2}\right)\right)
\end{aligned}
$$

with the Lagrange multiplier $\mu$.

We set the partial derivative of the last term w.r.t. $b_{12}$ to zero and solve it for $b_{12}$ to obtain

$$
\hat{b}_{12}(\mu)=\left(\Sigma_{\breve{b}_{12}}^{-1}+\mu 1\right)^{-1} \Sigma_{\breve{b}_{12}}^{-1} \breve{b}_{12}\left(N_{12}\right)
$$

Setting $\hat{b}_{12}(\mu)$ into the length constraint finally results in a root finding problem:

$$
f(\mu)=\left\|\hat{b}_{12}(\mu)\right\|^{2}-l^{2} \stackrel{!}{=} 0 .
$$

As the roots of $f(\mu)$ can not be found in closed form, we use the iterative Newton method. The Lagrange parameter $\mu$ is given at the $(n+1)$-th iteration by

$$
\mu^{(n+1)}=\mu^{(n)}-f(\mu) /\left.\frac{\partial}{\partial \mu} f(\mu)\right|_{\mu=\mu^{(n)}}
$$

with

$$
\frac{\partial}{\partial \mu} f(\mu)=2\left(\hat{b}_{12}(\mu)\right)^{\mathrm{T}} \frac{\partial}{\partial \mu}\left(\hat{b}_{12}(\mu)\right) .
$$

Let $\tilde{\Lambda}(\mu)=\Sigma_{z_{12}}^{-1}+\mu \cdot 1$, then

$$
\hat{b}_{12}(\mu)=(\tilde{\Lambda}(\mu))^{-1} \Sigma_{z_{12}}^{-1} z_{12}
$$

and

$$
\begin{aligned}
\frac{\partial}{\partial \mu}\left(\hat{b}_{12}(\mu)\right) & =\frac{\partial}{\partial \mu}\left(\tilde{\Lambda}^{-1}(\mu)\right) \Sigma_{z_{12}}^{-1} z_{12} \\
& =-\tilde{\Lambda}^{-1}(\mu) \frac{\partial}{\partial \mu}(\tilde{\Lambda}(\mu)) \tilde{\Lambda}^{-1}(\mu) \Sigma_{z_{12}}^{-1} z_{12} \\
& =-\tilde{\Lambda}^{-1}(\mu) \tilde{\Lambda}^{-1}(\mu) \Sigma_{z_{12}}^{-1} z_{12}
\end{aligned}
$$

\section{Measurement Analysis}

In this section, the proposed cycle slip detection and correction method is verified with real data. We used the following measurement setup:

- measurement period:

week number: 1738 , TOW $\in\{159,156 \mathrm{~s}, 159,812 \mathrm{~s}\}$

- measurement location (area of test drive):

longitude $\in\left\{11.41869^{\circ}, 11.42815^{\circ}\right\}$

latitude $\in\left\{47.99410^{\circ}, 47.99723^{\circ}\right\}$

- measurement equipment:

- 2 LEA 6T GPS receivers, $5 \mathrm{~Hz}$, u-blox

- 2 single frequency patch antennas, u-blox

- 1 MPU 9150 inertial sensor, $100 \mathrm{~Hz}$, Invensense

- installation:

- mounting of GPS antennas on roof of car

- alignment of baseline between antennas with longitudinal axis of car

- baseline length: $\bar{l}=1.32 \mathrm{~m}, \sigma_{\bar{l}}=1 \mathrm{~cm}$

Figure 2 shows selected sections of the test drive. In the first two sections, the car was passing below trees and between two buildings. The code and carrier phase signals of all visible satellites are temporarily affected by significant multipath. The last subfigure shows a slalom drive with high receiver dynamics.

Figure 3 shows the fixed double difference phase residuals before cycle slip detection and correction over time. In the upper left corner, a skyplot shows the satellite geometry. We applied an elevation mask of $20^{\circ}$. At 176, 241 and $401 \mathrm{~s}$, the car was passing below trees or between two buildings, which resulted in phase jumps of more than 10 half cycles. The largest residuals correspond to the satellites of lowest elevation. Numerous additional slips of $\{-2,-1,0,+1,+2\}$ cycles can be observed in between the major jumps.

Figure 4 shows the fixed double difference phase residuals after cycle slip detection and correction (CSC) using only GPS measurements in (23). The residuals are substantially lower than in Fig. 3 but there remain numerous undetected cycle slips.

Figure 5 shows the fixed double difference phase residuals after GPS/INS-based cycle slip detection and correction. The residuals of all satellites are substantially reduced to less than $15 \mathrm{~cm}$. Variations of the residuals of more than $1 \mathrm{~cm}$ correspond mainly to the double difference phase multipath. The subplot in the lower right corner shows the fixed phase residuals only for the three satellites of highest elevation. One can observe three sections with severe multipath. 
Fig. 2 Selected sections of the test drive: the first two sections are in a high multipath environment. The last section is characterized by high receiver dynamics. (a) 136-156 s: passing below trees, (b) 172-177 s: passing between two buildings, (c) 330-404 s: slalom drive
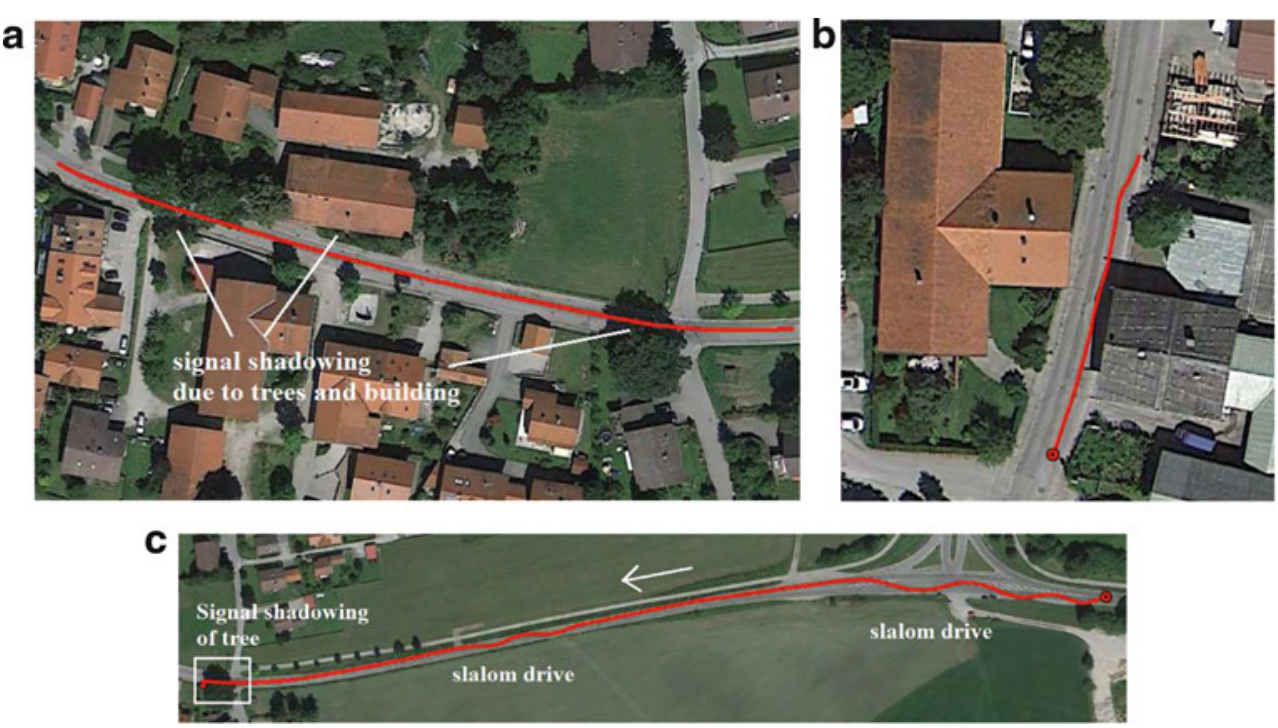

Fig. 3 Fixed phase residuals before cycle slip detection and correction: The residuals are jumping by more than 10 half cycles at 176, 241 and $401 \mathrm{~s}$, where the car was passing below trees or between two buildings. The largest residuals correspond to the satellites of lowest elevation. The satellite geometry is shown in the skyplot $\left(20^{\circ}\right.$ elevation mask) in the upper left corner. Numerous additional slips of $\{-2,-1,0,+1,+2\}$ cycles can be observed in between the major jumps

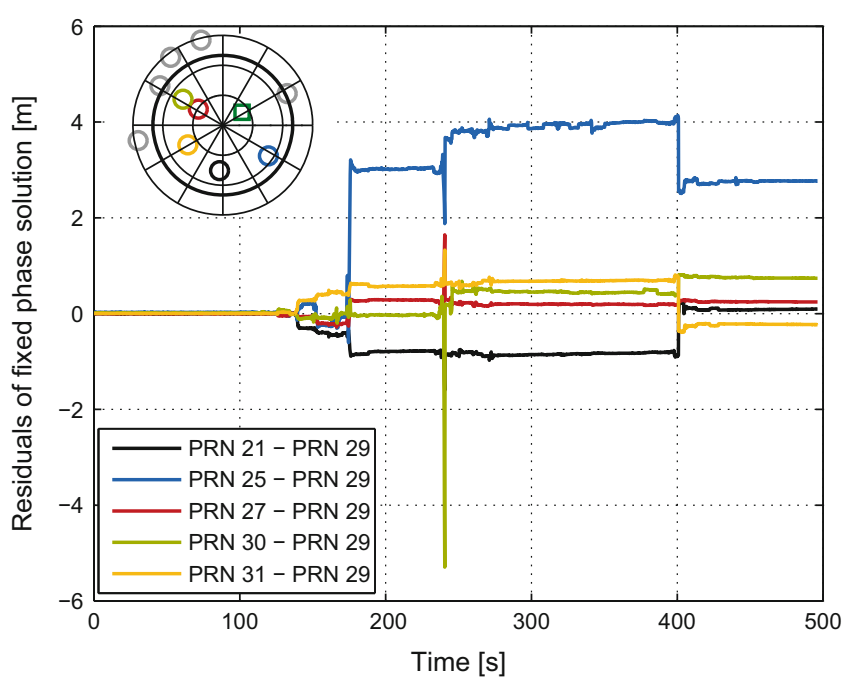

GPS measurement epoch after cycle slip correction with the GPS solution. Consequently, the drift of the IMU only between two subsequent GPS measurement epochs $(0.2 \mathrm{~s})$ is relevant. Figure 7 shows the difference between the heading of the IMU without continuous GPS-based calibration and the heading of the GPS/INS-combined solution. We can observe a slight continuous drift of less than $0.5^{\circ}$ with temporarily increased variations. The increased variations of up to $1^{\circ} / 0.2 \mathrm{~s}$ are most likely caused by heading errors of the GPS/INS-combined solution (in multipath environments) and not by changes of the IMU's drift. A cycle slip correction can still be performed reliably.

The proposed CSC of (27) jointly determines the baseline coordinates and integer cycle slips using both GPS DD carrier phases and the IMU-predicted baseline. It finds the optimized trade-off between minimizing the GPS measurement residuals and minimizing the IMU-predicted baseline residuals. We compare the performance of this optimized
The reliability of the GPS/INS combined CSC depends on the drift of the IMU. We re-initialize the IMU at every
GPS/INS CSC. In this section, the car was passing below a tree and all code and carrier phases were affected by substantial multipath. 
Fig. 4 Fixed phase residuals after GPS-based cycle slip detection and correction: the residuals are substantially reduced but there remain numerous undetected cycle slips

Fig. 5 Fixed phase residuals after GPS/IMU-based cycle slip detection and correction: the residuals of all satellites are reduced to less than $15 \mathrm{~cm}$. The "noise" in the residuals corresponds to the double difference phase multipath. The subplot in the lower right corner shows the fixed phase residuals only for the three satellites of highest elevation. One can observe three sections with severe multipath
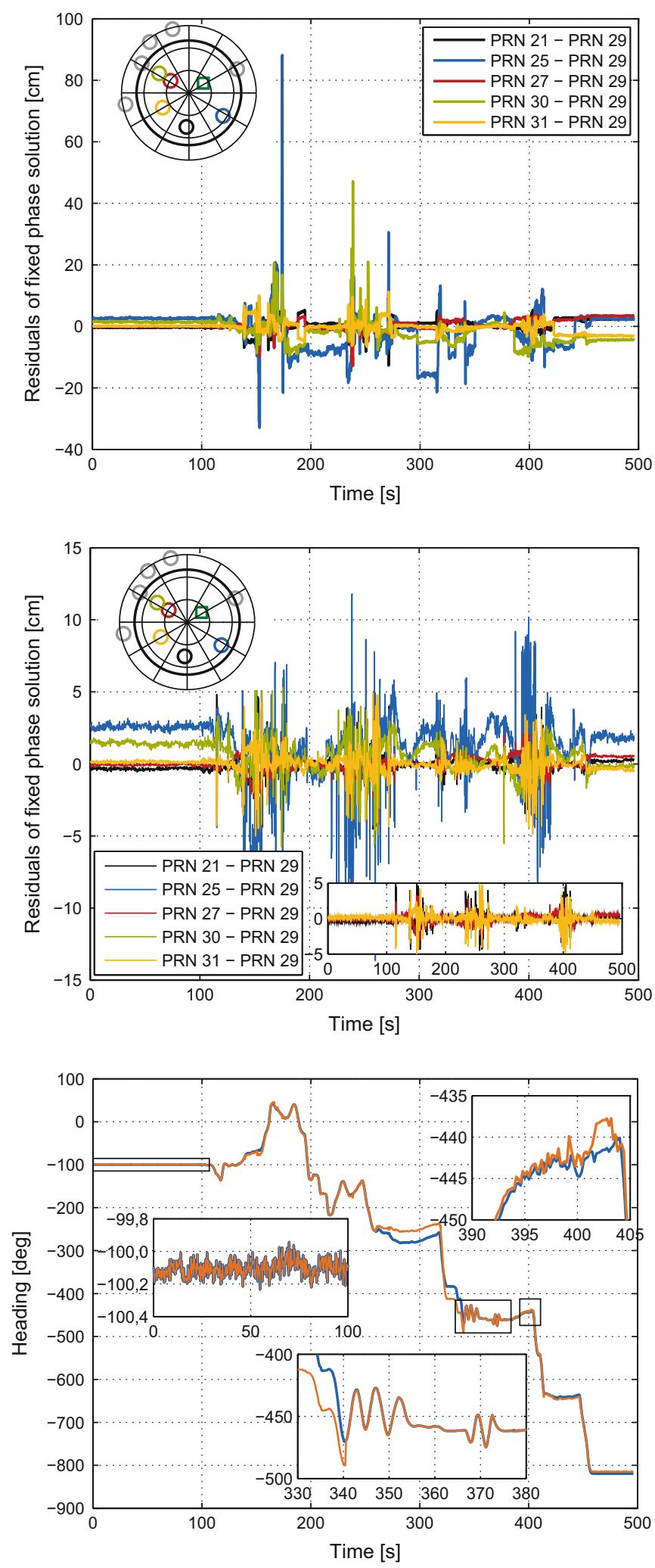

Fig. 6 Heading determination with GPS/INS combined cycle slip correction (marked in blue) and GPS-only (marked in orange) cycle slip correction: the noise level of both heading estimates is in the order of $0.1^{\circ}$ in static conditions and increases to $1^{\circ}$ in high multipath environments (e.g. passing below a tree between 395 and $405 \mathrm{~s}$ ). The periodic variations between 330 and $380 \mathrm{~s}$ indicate high receiver dynamics.

The GPS-based heading temporarily differs from the GPS/INS-based heading due to some uncorrected half cycle slips

approach to the performance of an integer least-squares CSC estimation with the baseline vector considered known and fixed from the IMU. This alternative approach also combines GPS and INS measurements. However, the integer least- squares estimation only of the CSC does not guarantee a final baseline estimate to be close to the one from the IMU. Figure 8 shows that the heading of the integer leastsquares estimation can differ by up to $20^{\circ}$ from the combined 
Fig. 7 Drift of heading of inertial sensor without continuous GPS-based calibration: the change of the heading between two subsequent epochs is in general less than $0.5^{\circ}$. The largest variation of $1^{\circ} / 0.2 \mathrm{~s}$ occurs during the passing below trees, where the coupled GPS/INS was itself more noisy. A cycle slip correction can still be performed reliably

Fig. 8 Cycle slip correction with GPS/INS: the heading of the integer least-squares cycle slip estimation with fixed IMU baseline differs by up to $20^{\circ}$ from the heading of the combined cycle slip and baseline estimation using both GPS and IMU measurements. This indicates that a pure integer least-squares estimation is not sufficient. As the combined estimator finds an optimized trade-off between minimizing the squared measurement residuals and minimizing the baseline vector residuals, it finds the correct cycle slip correction with highest success rate

solution. This shows again the need for the proposed cycle slip detection and correction method, which combines all available measurement and a priori information and minimizes the sum of the measurement residuals and a priori information residuals.

The reliability of cycle slip detection and correction can also be verified by adding artificial cycle slips to the raw phase measurements. We have tested all $\{-2,-1,0,+1,2\}$ cycle slip combinations for $\{1,2,3\}$ simultaneous cycle slips at a moment of high receiver dynamics $(t=342 \mathrm{~s})$, and observed that all cycle slip combinations were correctly found.

\section{Conclusion}

In this paper, a method for reliable cycle slip detection and correction was proposed for low-cost GPS receivers with high receiver dynamics in challenging environments. It combines double difference GPS carrier phases from all visible
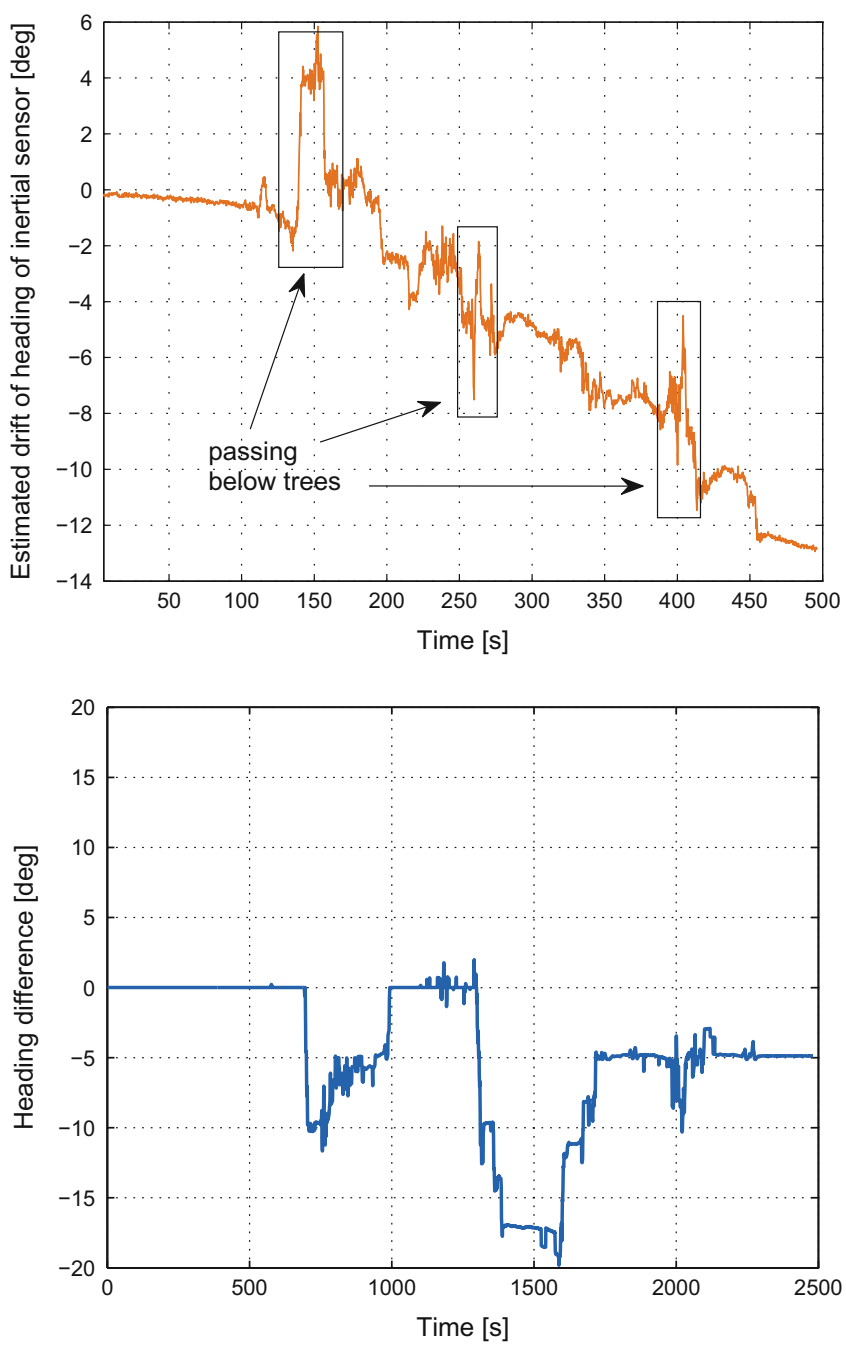

satellites, gyroscope and acceleration measurements, and a priori information on the baseline length. It performs a tree search for finding the cycle slip corrections. The estimator was first tested by a simulation of artificial cycle slips. It turned out to be extremely powerful if the IMU was properly calibrated, i.e. it was able to correct simultaneous cycle slips at all visible satellites. Subsequently, the proposed method was verified in a test drive with two low-cost GPS receivers and a low-cost 9-axes INS. The residuals of the fixed phase measurements after cycle slip correction remained less than $15 \mathrm{~cm}$ in both a slalom drive with high receiver dynamics and passages below trees.

\section{References}

Colombo OL, Bhapkar UV, Evans AG (1999) Inertial-aided cycle-slip detection/correction for precise, long-baseline kinematic GPS. In: Proceedings of the 12th ION GNSS, Nashville, pp 1915-1922

Dai Z, Knedlik S, Loffeld O (2009) Instantaneous triple-frequency GPS cycle-slip detection and repair. Intl J Navig Obs 2009:15. Article ID 407231 
Du S, Gao Y (2012) Inertial aided cycle slip detection and identification for integrated PPP GPS and INS. Sensors 12(11):14,344-14,362

Henkel P, Gunther C (2013) Attitude determination with low-cost GPS/INS. In: Proceedings of the 26-th ION GNSS+, Nashville, pp 2015-2023

Henkel P, Kiam JJ (2013) Maximum a posteriori probability estimation of integer ambiguities and baseline. In: IEEE proceedings of the 55th symposium ELMAR, Zadar, pp 353-356
Jekeli C (2001) Inertial navigation systems with geodetic applications. Walter de Gruyter

Teunissen P (1995) The least-squares ambiguity decorrelation adjustment: a method for fast GPS ambiguity estimation. J Geod 70:65-82

Teunissen P (2006) The LAMBDA method for the GNSS compass. Artif Satell 41(3):89-103

Teunissen P (2010) Integer least-squares theory for the GNSS compass. J Geod 84:433-447 